\title{
Adaptive Radial Basis Function Detector for Beamforming
}

\author{
Sheng Chen, Khaled Labib, Rong Kang and Lajos Hanzo \\ School of Electronics and Computer Science \\ University of Southampton, Southampton SO17 1BJ, UK \\ E-mails: $\{$ sqc,koml105,rk405,lh\}@ecs.soton.ac.uk
}

\begin{abstract}
We consider nonlinear detection in rank-deficient multiple-antenna assisted beamforming systems. By exploiting the inherent symmetry of the underlying optimal Bayesian detection solution, a symmetric radial basis function (RBF) detector is proposed and two adaptive algorithms are developed for training the proposed RBF detector. The first adaptive algorithm, referred to as the nonlinear least bit error, is a stochastic approximation to the Parzen window estimation of the detector output's probability density function while the second algorithm is based on a clustering. The proposed adaptive solutions are capable of providing a signal to noise ratio gain in excess of $8 \mathrm{~dB}$ against the theoretical linear minimum bit error rate benchmarker, when supporting four users with the aid of two receive antennas or five users employing three antenna elements.
\end{abstract}

\section{INTRODUCTION}

Adaptive beamforming is capable of separating user signals transmitted on the same carrier frequency, and thus provides a practical means of supporting multiusers in a space-division multiple-access scenario [1]-[10]. Classically, this is achieved by a linear beamformer based on the minimum mean square error (L-MMSE) solution [1],[2],[7],[8],[11]. The L-MMSE beamforming requires that the number of users supported is no more than the number of receive antenna elements. If this condition is not met, the system is referred to as overloaded or rank-deficient. The optimal solution for the linear beamforming has been shown to be the minimum bit error rate (L-MBER) design [12],[13] which outperforms the L-MMSE one and is capable of operating in hostile rankdeficient scenarios. However, digital communication signal detection can be viewed as a classification problem [14][16], where the receiver detector simply classifies the received multidimensional channel-impaired signal into the most-likely transmitted symbol constellation point or class. Both the radial basis function (RBF) network [17]-[19] and other kernel models [20]-[25] have been applied to solve this nonlinear detection problem. All these nonlinear detectors attempt to approximate the underlying optimal Bayesian solution.

The standard RBF or kernel modelling technique constitutes a black-box approach that seeks to extract a model representation from the available training data. Adopting this black-box modelling approach is appropriate, if no a priori information exists regarding the underlying data generating mechanism. If however there exists some a priori information concerning the system to be modelled, this a priori information should be incorporated into the modelling process. Many real-life phenomena exhibit inherent properties, such as symmetry, but these properties are often hard to infer from the data with the aid of black-box models. In regression modelling, the symmetric properties of the underlying system have been exploited by imposing symmetry in both RBF networks and least squares support vector machines [26],[27]. By imposing symmetry on the model's structure, it is easier to extract the inherent symmetry properties of the underlying system from noisy training data and this leads to substantial improvements in the achievable regression modelling performance. In this paper, we propose a symmetric RBF detector for multipleantenna aided beamforming systems.

The optimal Bayesian nonlinear detection solution has an inherent symmetry because the signal states corresponding to the different signal classes are distributed symmetrically. This symmetric property is difficult to infer from noisy training data using a standard RBF model. In fact, previous studies [16]-[25] have shown that a standard RBF detector typically requires significantly more RBF centres than the number of channel output states in order to approximate the Bayesian detector using noisy training data, and often there is a performance difference between such a RBF detector and the optimal Baysian solution. In contrast to the standard RBF model, the proposed symmetric RBF model is capable of approaching the optimal Bayesian performance accurately, despite using channel-impaired training data and despite using no more RBF centres than the number of channel output states. The advantage of the proposed symmetric RBF detector is demonstrated in challenging detection scenarios, where the number of users supported is almost twice the number of antenna array elements. Two adaptive algorithms are developed to update the symmetric RBF detector's parameters on a sampleby-sample basis for the sake of maintaining a low real-time computational complexity. The first adaptive algorithm is a stochastic learning algorithm, referred to as the nonlinear least bit error rate (NLBER), which directly minimises an approximate detection error probability or bit error rate (BER). The second adaptive algorithm is based on the enhanced $\kappa$ means clustering algorithm [16].

\section{Multiple Antenna Assisted Beamforming}

The system supports $M$ users, where each user transmits on the same carrier frequency of $\omega=2 \pi f$, and the receiver is equipped with a linear antenna array consisting of $L$ 
uniformly spaced elements. Further assume that the channel is non-dispersive. Then the symbol-rate complex-valued received signal samples can be expressed as

$$
x_{l}(k)=\sum_{i=1}^{M} A_{i} b_{i}(k) e^{j \omega t_{l}\left(\theta_{i}\right)}+n_{l}(k)=\bar{x}_{l}(k)+n_{l}(k),
$$

for $1 \leq l \leq L$, where $t_{l}\left(\theta_{i}\right)$ is the relative time delay at array element $l$ for source $i$, with $\theta_{i}$ being the direction of arrival for source $i, n_{l}(k)$ is the Gaussian white noise with $E\left[\left|n_{l}(k)\right|^{2}\right]=2 \sigma_{n}^{2}, A_{i}$ is the channel coefficient of user $i$, and $b_{i}(k)$ is the $k$-th symbol of user $i$, taking values from a binary phase shift keying (BPSK) symbol set, i.e. $b_{i}(k) \in\{ \pm 1\}$. Source 1 is the desired user and the rest of the sources are the interfering users. The desired user's signal-to-noise ratio is given by $\mathrm{SNR}=\left|A_{1}\right|^{2} \sigma_{b}^{2} / 2 \sigma_{n}^{2}$, where $\sigma_{b}^{2}=1$ is the BPSK symbol energy, and the desired signal-to-interferer $i$ ratio is defined by $\operatorname{SIR}_{i}=\left|A_{1}\right|^{2} /\left|A_{i}\right|^{2}$, for $2 \leq i \leq M$. The received signal vector $\mathbf{x}(k)=\left[x_{1}(k) \cdots x_{L}(k)\right]^{T}$ can be expressed as

$$
\mathbf{x}(k)=\mathbf{P b}(k)+\mathbf{n}(k)=\overline{\mathbf{x}}(k)+\mathbf{n}(k),
$$

where $\mathbf{n}(k)=\left[n_{1}(k) \cdots n_{L}(k)\right]^{T}$ and the system matrix $\mathbf{P}$ is given by $\mathbf{P}=\left[A_{1} \mathbf{s}_{1} \cdots A_{M} \mathbf{s}_{M}\right]$ with the steering vector of source $i$ given by $\mathbf{s}_{i}=\left[e^{j \omega t_{1}\left(\theta_{i}\right)} \cdots e^{j \omega t_{L}\left(\theta_{i}\right)}\right]^{T}$, and the transmitted BPSK symbol vector by $\mathbf{b}(k)=\left[b_{1}(k) \cdots b_{M}(k)\right]^{T}$. Traditionally, a linear beamforming receiver, $y_{\operatorname{Lin}}(k)=$ $\boldsymbol{\theta}^{T} \mathbf{x}(k)$, is adopted to detect the desired user's signal [1],[7] with the associated decision given by

$$
\hat{b}_{1}(k)=\operatorname{sgn}\left(\Re\left[y_{\operatorname{Lin}}(k)\right]\right)= \begin{cases}+1, & \Re\left[y_{\text {Lin }}(k)\right] \geq 0, \\ -1, & \Re\left[y_{\text {Lin }}(k)\right]<0,\end{cases}
$$

where $\boldsymbol{\theta}=\left[\theta_{1} \cdots \theta_{L}\right]^{T}$ denotes the complex-valued linear beamformer's weight vector and $\Re[\bullet]$ the real part. Classically, the L-MMSE solution for the weight vector of the linear beamformer is regarded as the optimal design [1],[2],[7],[8],[11]. The L-MMSE technique requires that the number of users $M$ is no higher than the number of antenna array elements $L$. The optimal weight vector designed for the linear beamformer is known to be the L-MBER solution [12],[13] which directly minimises the BER of the linear beamformer and is capable of operating in rank-deficient scenarios.

However, the optimal multiple antenna aided beamforming detector is nonlinear [16],[25]. Let us denote the $N_{b}=2^{M}$ combinations of $\mathbf{b}(k)$ as $\mathbf{b}_{q}, 1 \leq q \leq N_{b}$, and the first element of $\mathbf{b}_{q}$, related to the desired user, as $b_{q, 1}$. The noiseless channel output $\overline{\mathbf{x}}(k)$ takes values from the finite signal set $\overline{\mathbf{x}}(k) \in \mathcal{X} \triangleq\left\{\overline{\mathbf{x}}_{q}=\mathbf{P b}_{q}, 1 \leq q \leq N_{b}\right\}$, which can be divided into two subsets conditioned on the value of $b_{1}(k)$ as

$$
\mathcal{X}^{( \pm)} \triangleq\left\{\overline{\mathbf{x}}_{i} \in \mathcal{X}, 1 \leq i \leq N_{s b}: b_{1}(k)= \pm 1\right\},
$$

where the size of $\mathcal{X}^{(+)}$and $\mathcal{X}^{(-)}$is $N_{s b}=N_{b} / 2=2^{M-1}$. Let the conditional probabilities of receiving $\mathbf{x}(k)$ given $b_{1}(k)=$ \pm 1 be $p_{ \pm}(\mathbf{x}(k))=p\left(\mathbf{x}(k) \mid b_{1}(k)= \pm 1\right)$. According to Bayes decision theory [28], the optimal detection strategy is

$$
\hat{b}_{1}(k)= \begin{cases}+1, & \text { if } \quad p_{+}(\mathbf{x}(k)) \geq p_{-}(\mathbf{x}(k)), \\ -1, & \text { if } \quad p_{+}(\mathbf{x}(k))<p_{-}(\mathbf{x}(k)) .\end{cases}
$$

If we introduce the real-valued Bayesian decision variable

$$
y_{\text {Bay }}(k)=f_{\text {Bay }}(\mathbf{x}(k)) \triangleq \frac{1}{2} p_{+}(\mathbf{x}(k))-\frac{1}{2} p_{-}(\mathbf{x}(k)),
$$

the optimal detection rule (5) is equivalent to $\hat{b}_{1}(k)=$ $\operatorname{sgn}\left(y_{\text {Bay }}(k)\right)$. Decision variable (6) can be expressed as

$$
y_{\text {Bay }}(k)=\sum_{q=1}^{N_{b}} \operatorname{sgn}\left(b_{q, 1}\right) \beta_{q} e^{-\frac{\left\|\mathbf{x}(k)-\overline{\mathbf{x}}_{q}\right\|^{2}}{2 \sigma_{n}^{2}}}
$$

where $\beta_{q}$ denotes the a priori probability of $\overline{\mathbf{x}}_{q}$. Since all the $\overline{\mathbf{x}}_{q}$ are equiprobable, $\beta_{q}=\beta=\frac{1}{N_{b}\left(2 \pi \sigma_{n}^{2}\right)^{L}}$. The two subsets $\mathcal{X}^{(+)}$and $\mathcal{X}^{(-)}$are distributed symmetrically, namely, for any signal state $\overline{\mathbf{x}}_{i}^{(+)} \in \mathcal{X}^{(+)}$there exists a signal state $\overline{\mathbf{x}}_{i}^{(-)} \in \mathcal{X}^{(-)}$satisfying $\overline{\mathbf{x}}_{i}^{(-)}=-\overline{\mathbf{x}}_{i}^{(+)}$. Given this symmetry, the optimal Bayesian detector (7) can be characterised as

$$
y_{\text {Bay }}(k)=\sum_{q=1}^{N_{s b}} \beta_{q}\left(e^{-\frac{\left\|\mathbf{x}(k)-\overline{\mathbf{x}}_{q}^{(+)}\right\|^{2}}{2 \sigma_{n}^{2}}}-e^{-\frac{\left\|\mathbf{x}(k)+\overline{\mathbf{x}}_{q}^{(+)}\right\|^{2}}{2 \sigma_{n}^{2}}}\right),
$$

where $\overline{\mathbf{x}}_{q}^{(+)} \in \mathcal{X}^{(+)}$. The Bayesian detector has odd symmetry, as $f_{\text {Bay }}(-\mathbf{x}(k))=-f_{\text {Bay }}(\mathbf{x}(k))$. This symmetry is hard to infer from the noisy data by a standard RBF detector.

\section{Symmetric RAdial Basis Function Detector}

Consider the training of the RBF detector of the form

$$
y_{\mathrm{RBF}}(k)=f_{\mathrm{RBF}}(\mathbf{x}(k) ; \mathbf{w})=\sum_{i=1}^{n_{c}} \theta_{i} \phi_{i}(\mathbf{x}(k)),
$$

based on the training data set $D_{K}=\left\{\mathbf{x}(k), b_{1}(k)\right\}_{k=1}^{K}$, where $f(\bullet ; \bullet)$ is a real-valued nonlinear mapping realised by the RBF network, $\theta_{i}$ is the $i$ th real-valued RBF weight, $\phi_{i}(\bullet)$ denotes the response of the $i$-th RBF node, $n_{c}$ is the number of RBF nodes used, and $\mathrm{w}$ denotes the vector of all the adjustable parameters of the RBF detector. We propose to adopt the following symmetric RBF node

$$
\phi_{i}(\mathbf{x}) \triangleq \varphi\left(\mathbf{x} ; \mathbf{c}_{i}, \sigma_{i}^{2}\right)-\varphi\left(\mathbf{x} ;-\mathbf{c}_{i}, \sigma_{i}^{2}\right),
$$

where $\mathbf{c}_{i}$ is the $i$ th complex-valued RBF centre, $\sigma_{i}^{2}$ the $i$ th real-valued RBF variance, and $\varphi(\bullet)$ the classic RBF function. In this study we adopt the Gaussian RBF function

$$
\varphi\left(\mathbf{x} ; \mathbf{c}_{i}, \sigma^{2}\right)=e^{-\frac{\left\|\mathbf{x}-\mathbf{c}_{i}\right\|^{2}}{\sigma^{2}}} .
$$

The symmetric RBF network (9) with the node structure (10) has an inherently odd symmetry. A standard RBF model with the RBF node defined by $\phi_{i}(\mathbf{x}) \triangleq \varphi\left(\mathbf{x} ; \mathbf{c}_{i}, \sigma_{i}^{2}\right)$, by contrast, cannot guarantee odd symmetry, particularly when the RBF centres are generated from noisy training data.

\section{A. The nonlinear least bit error rate algorithm}

Let us define the signed decision variable $y_{s}(k)=$ $\operatorname{sgn}\left(b_{1}(k)\right) y_{\mathrm{RBF}}(k)$ and denote the probability density function (PDF) of $y_{s}(k)$ as $p_{y}\left(y_{s}\right)$. Then the error probability of the nonlinear detector (9) is given by

$$
P_{E}(\mathbf{w})=\operatorname{Prob}\left\{y_{s}(k)<0\right\}=\int_{-\infty}^{0} p_{y}\left(y_{s}\right) d y_{s} .
$$


The MBER solution for the detector's parameter vector $\mathbf{w}$ is defined as the one that minimises $P_{E}(\mathbf{w})$. The problem with this approach is that the PDF of $y_{s}(k)$ is unknown. However, it may be sufficiently accurately estimated using the Parzen window method [29]-[31]. Given a block of training data $D_{K}$, a Parzen window estimate of $p_{y}\left(y_{s}\right)$ is readily given as

$$
\tilde{p}_{y}\left(y_{s}\right)=\frac{1}{K \sqrt{2 \pi} \rho} \sum_{k=1}^{K} e^{-\frac{\left(y_{s}-\operatorname{sgn}_{\left.\left(b_{1}(k)\right) y_{\mathrm{RBF}}(k)\right)^{2}}^{2 \rho^{2}}\right.}{},}
$$

where $\rho^{2}$ is the chosen kernel variance. With this estimated PDF, the estimated or approximate BER is given by

$$
\tilde{P}_{E}(\mathbf{w})=\int_{-\infty}^{0} \tilde{p}_{y}\left(y_{s}\right) d y_{s}=\frac{1}{K} \sum_{k=1}^{K} Q\left(\tilde{g}_{k}(\mathbf{w})\right),
$$

where $Q(u)$ is the usual Gaussian error function and $\tilde{g}_{k}(\mathbf{w})=$ $\operatorname{sgn}\left(b_{1}(k)\right) y_{\mathrm{RBF}}(k) / \rho$. An approximate MBER solution for $\mathbf{w}$ can be obtained by minimising $\tilde{P}_{E}(\mathbf{w})$.

In order to derive a sample-by-sample adaptive algorithm, consider a "single-sample PDF estimate" of $p_{y}\left(y_{s}\right)$ given by

$$
\tilde{p}_{y}\left(y_{s}, k\right)=\frac{1}{\sqrt{2 \pi} \rho} e^{-\frac{\left(y_{s}-\operatorname{Sgn}_{\left.\left(b_{1}(k)\right) y_{\mathrm{RBF}}(k)\right)^{2}}\right.}{2 \rho^{2}}} .
$$

Conceptually, given this instantaneous PDF "estimate" we have a single-sample BER "estimate" $\tilde{P}_{E}(\mathbf{w}, k)=Q\left(\tilde{g}_{k}(\mathbf{w})\right)$. Using the instantaneous gradient $\nabla \tilde{P}_{E}(\mathbf{w}, k)$ gives rise to the following stochastic adaptive algorithm

$$
\begin{aligned}
\mathbf{w}(k)= & \mathbf{w}(k-1)+\frac{\mu}{\sqrt{2 \pi} \rho} e^{-\frac{y_{\mathrm{RBF}}^{2}(k)}{2 \rho^{2}}} \operatorname{sgn}\left(b_{1}(k)\right) \\
& \times \frac{\partial f_{\mathrm{RBF}}(\mathbf{x}(k) ; \mathbf{w}(k-1))}{\partial \mathbf{w}},
\end{aligned}
$$

which we refer to as the NLBER algorithm. The step size $\mu$ and kernel variance $\rho^{2}$ should be chosen appropriately to achieve a desired convergence performance, both in terms of convergence speed and steady-state BER misadjustment. For the symmetric RBF detector (9) using the Gaussian RBF function of (11), the derivatives of the RBF detector's output with respect to the RBF detector's parameters are given by

$$
\begin{aligned}
& \frac{\partial f_{\mathrm{RBF}}}{\partial \theta_{i}}=e^{-\frac{\left\|\mathbf{x}(k)-\mathbf{c}_{i}\right\|^{2}}{\sigma_{i}^{2}}}-e^{-\frac{\left\|\mathbf{x}(k)+\mathbf{c}_{i}\right\|^{2}}{\sigma_{i}^{2}}}, \\
& \frac{\partial f_{\mathrm{RBF}}}{\partial \sigma_{i}^{2}}=\theta_{i}\left(e^{-\frac{\left\|\mathbf{x}(k)-\mathbf{c}_{i}\right\|^{2}}{\sigma_{i}^{2}}} \frac{\left\|\mathbf{x}(k)-\mathbf{c}_{i}\right\|^{2}}{\left(\sigma_{i}^{2}\right)^{2}}-e^{-\frac{\left\|\mathbf{x}(k)+\mathbf{c}_{i}\right\|^{2}}{\sigma_{i}^{2}}} \frac{\left\|\mathbf{x}(k)+\mathbf{c}_{i}\right\|^{2}}{\left(\sigma_{i}^{2}\right)^{2}}\right), \\
& \frac{\partial f_{\mathrm{RBF}}}{\partial \mathbf{c}_{i}}=\theta_{i}\left(e^{-\frac{\left\|\mathbf{x}(k)-\mathbf{c}_{i}\right\|^{2}}{\sigma_{i}^{2}}} \frac{\mathbf{x}(k)-\mathbf{c}_{i}}{\sigma_{i}^{2}}+e^{-\frac{\left\|\mathbf{x}(k)+\mathbf{c}_{i}\right\|^{2}}{\sigma_{i}^{2}}} \frac{\mathbf{x}(k)+\mathbf{c}_{i}}{\sigma_{i}^{2}}\right) .
\end{aligned}
$$

\section{B. The clustering algorithm}

Let $\hat{\sigma}_{n}^{2}$ be an estimated $\sigma_{n}^{2}$ and set all the RBF variances

\begin{tabular}{|c|c|c|c|c|c|}
\hline \multicolumn{6}{|c|}{ Example 1: two-element antenna array supporting four users } \\
\hline user $i$ & 1 & 2 & 3 & 4 & \\
\hline AOA $\theta$ & $0^{\circ}$ & $20^{\circ}$ & $-30^{\circ}$ & $-45^{\circ}$ & \\
\hline \multicolumn{6}{|c|}{ Example 2: three-element antenna array supporting five users } \\
\hline user $i$ & 1 & 2 & 3 & 4 & 5 \\
\hline AOA $\theta$ & $0^{\circ}$ & $10^{\circ}$ & $-17^{\circ}$ & $15^{\circ}$ & $20^{\circ}$ \\
\hline
\end{tabular}
$\sigma_{i}^{2}=2 \hat{\sigma}_{n}^{2}$. Further assume that $n_{c}=N_{s b}$ and set all the RBF weights $\beta_{i}$ to a same positive value. Then in order for the symmetric RBF detector (9) to realise the Bayesian solution, we have to choose the RBF centres $\mathbf{c}_{i}, 1 \leq i \leq N_{s b}$,
TABLE I

NUMBER OF ANTENNA ARRAY ELEMENTS, NUMBER OF USERS SUPPORTED AND LOCATIONS OF USERS IN TERMS OF ANGLE OF ARRIVAL (AOA).

appropriately. This task can be achieved by the enhanced $\kappa$ means clustering algorithm [16]. Note that unlike in the singleuser case, the receiver only has access to $b_{1}(k)$, not $\mathbf{b}(k)$, during training, and the following clustering algorithm can be used to update the RBF centres

$$
\mathbf{c}_{i}(k)=\mathbf{c}_{i}(k-1)+\mu_{c} \mathcal{M}_{i}(\check{\mathbf{x}}(k))\left(\check{\mathbf{x}}(k)-\mathbf{c}_{i}(k-1)\right),
$$

where $\check{\mathbf{x}}(k)=\mathbf{x}(k)$ if $b_{1}(k)=+1, \check{\mathbf{x}}(k)=-\mathbf{x}(k)$ if $b_{1}(k)=$ -1 , and $\mu_{c}$ is the step size. The membership function $\mathcal{M}_{i}(\mathbf{x})$ is defined as

$$
\mathcal{M}_{i}(\mathbf{x})= \begin{cases}1, & \text { if } \bar{v}_{i}\left\|\mathbf{x}-\mathbf{c}_{i}\right\|^{2} \leq \bar{v}_{j}\left\|\mathbf{x}-\mathbf{c}_{j}\right\|^{2}, \forall j \neq i \\ 0, & \text { otherwise }\end{cases}
$$

where $\bar{v}_{i}$ is the variation of the $i$ th cluster. In order to estimate the associated variation $\bar{v}_{i}$, the following updating rule is used

$\bar{v}_{i}(k)=\mu_{v} \bar{v}_{i}(k-1)+\left(1-\mu_{v}\right) \mathcal{M}_{i}(\check{\mathbf{x}}(k))\left\|\check{\mathbf{x}}(k)-\mathbf{c}_{i}(k-1)\right\|^{2}$,

where $\mu_{v}$ is a constant slightly less than 1.0. The initial variations $\bar{v}_{i}(0), \forall i$, are set to the same small number.

\section{Simulation STUdY}

Two simulated beamforming systems, as listed in Table I, were used. The array element spacing was half of the wavelength. The simulated narrowband channels were $A_{i}=1+j 0,1 \leq$ $i \leq M$, and the desired user and all the interfering users had equal signal power. Therefore $\mathrm{SIR}_{i}=0 \mathrm{~dB}$ for $2 \leq i \leq M$.

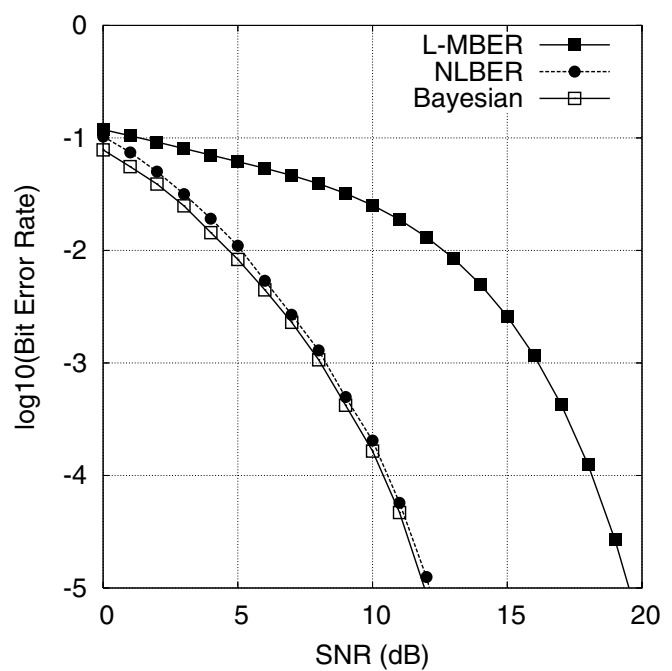

Fig. 1. The desired-user's bit error rate performance for the two-element antenna array supporting four users at the angular positions of Table I. The NLBER-based RBF detector has $n_{c}=8$ symmetric RBF nodes. 


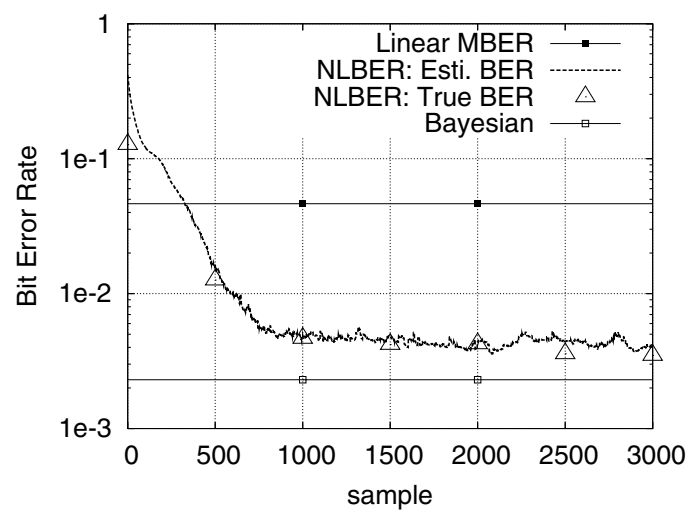

Fig. 2. Learning curve of the NLBER-based RBF detector averaged over 10 runs for the two-element antenna array supporting four users, where SNR= $7 \mathrm{~dB}$ and the RBF detector has $n_{c}=8$ symmetric RBF nodes.

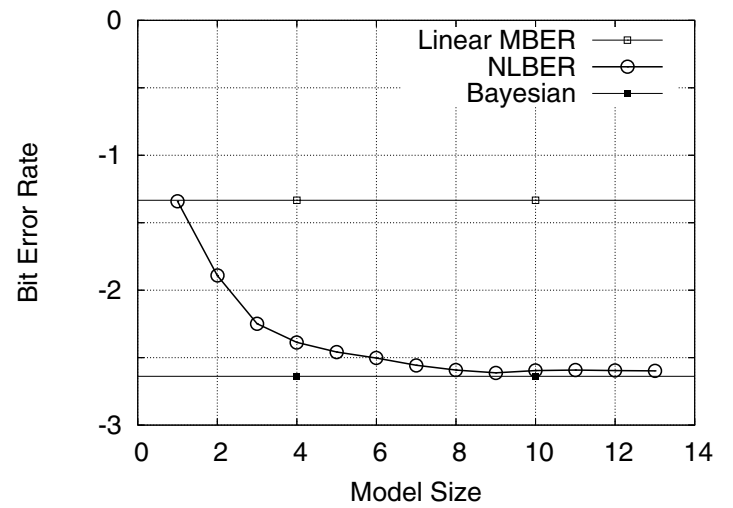

Fig. 3. The influence of the detector's size on the bit error rate performance of the NLBER-based symmetric RBF detector for the two-element antenna array supporting four users, where $\mathrm{SNR}=7 \mathrm{~dB}$.

Example 1. Fig. 1 depicts the BERs of both the L-MBER beamformer and the Bayesian detector for the desired user. The size of the Bayesian detector is specified by the number of symmetric signal states $N_{s b}=8$. Given $\mathrm{SNR}=7 \mathrm{~dB}$ and detector size $n_{c}=8$, Fig. 2 shows the learning curve of the NLBER algorithm averaged over 10 runs, where we used the first eight data points as the initial $\mathrm{RBF}$ centres $\mathbf{c}_{i}(0)$, set the initial RBF weights to $\theta_{i}(0)=0.1$ and the initial $\mathrm{RBF}$ variances to $\sigma_{i}^{2}(0)=\sigma_{n}^{2}$, for $1 \leq i \leq n_{c}=8$. The step size and kernel variance of the NLBER algorithm (16) were chosen to be $\mu=0.4$ and $\rho^{2}=10 \sigma_{n}^{2}$. These values were found empirically to be appropriate. The learning curve (dashed curve) was the estimated BER $\tilde{P}_{E}(\mathbf{w}(k))$, calculated using (14) with a block size of $K=400$ and a kernel variance of $\rho^{2}=\sigma_{n}^{2}$. To check that the estimated BER $\tilde{P}_{E}(\mathbf{w}(k))$ gave the correct convergence trend, we also calculated the true BER $P_{E}(\mathbf{w}(k))$ using Monte Carlo simulation for a number of points, shown in Fig. 2 by the triangles. With the same initial conditions, Fig. 3 illustrates the performance of the NLBER-based detector as a function of model size $n_{c}$. For $n_{c} \geq N_{s b}$ the symmetric RBF detector trained by the stochastic NLBER algorithm is capable of closely approaching the optimal Bayesian performance. The BER of the NLBERbased symmetric RBF detector having $n_{c}=8 \mathrm{RBF}$ nodes is depicted in Fig. 1, in comparison to the Bayesian detector.

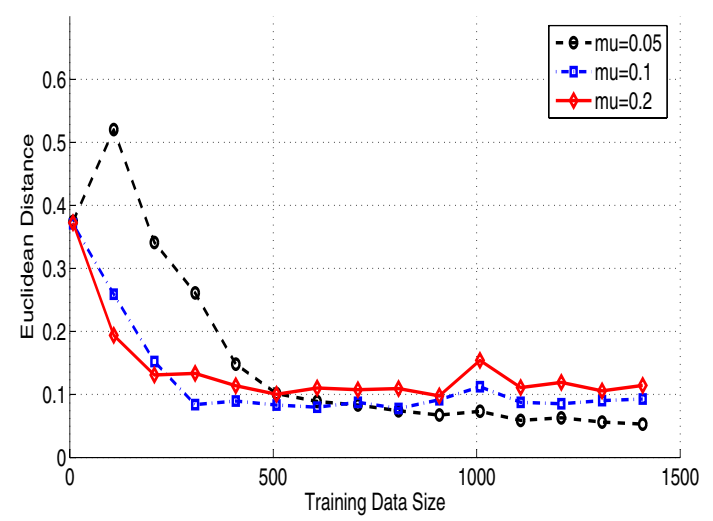

Fig. 4. Learning curve of the clustering-based RBF detector averaged over 5 runs for the two-element antenna array supporting four users, where SNR= $7 \mathrm{~dB}$ and the RBF detector has $n_{c}=8$ symmetric RBF nodes.

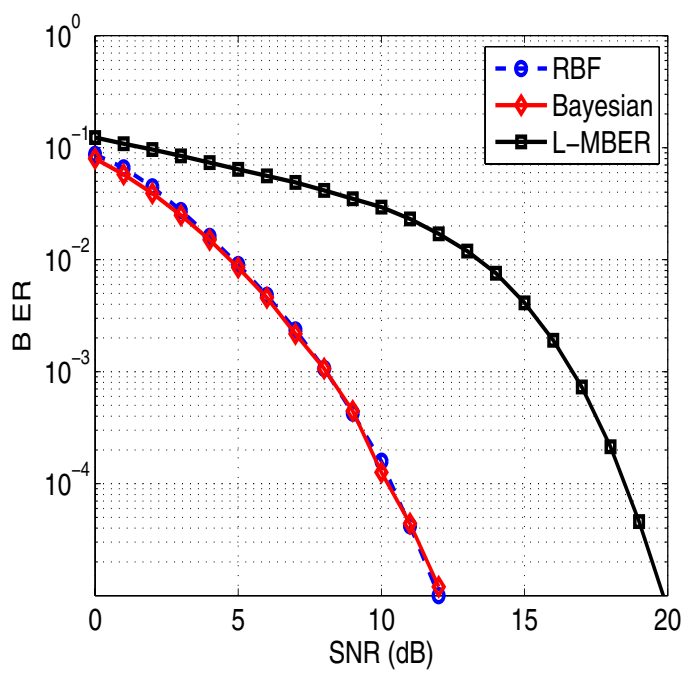

Fig. 5. The desired-user's bit error rate performance for the two-element antenna array supporting four users at the angular positions of Table I. The clustering-based RBF detector has $n_{c}=8$ symmetric RBF nodes.

The clustering-based symmetric RBF detector was next investigated. We used the first $N_{s b}$ data points as the initial centres $\mathbf{c}_{i}(0)$ and set all the RBF variances to $2 \sigma_{n}^{2}$. The convergence of the clustering algorithm (17) was assessed based on the following Euclidean distance metric

$$
\operatorname{ED}(k)=\sum_{i=1}^{N_{s b}}\left\|\mathbf{c}_{i}(k)-\overline{\mathbf{x}}_{i}^{(+)}\right\|^{2} .
$$

The initial clustering variations $\bar{v}_{i}(0)$ were set to 0.01 and the adaptive gain for updating the clustering variations was set to $\mu_{v}=0.995$. Fig. 4 depicts the learning curves of the clustering algorithm averaged over 5 runs for different values of step size $\mu_{c}$. The BER of the clustering-based symmetric RBF detector is plotted in Fig. 5, in comparison with the two benchmark detectors. We also studied the influence of the RBF variance. For the same conditions in Fig. 4, it was observed that the RBF detector achieved the Bayesian performance given the RBF variance in the range of $0.1 \times 2 \sigma_{n}^{2}$ to $10 \times 2 \sigma_{n}^{2}$. This confirms that the performance of the RBF detector is insensitive to the value of its $R B F$ variance. 


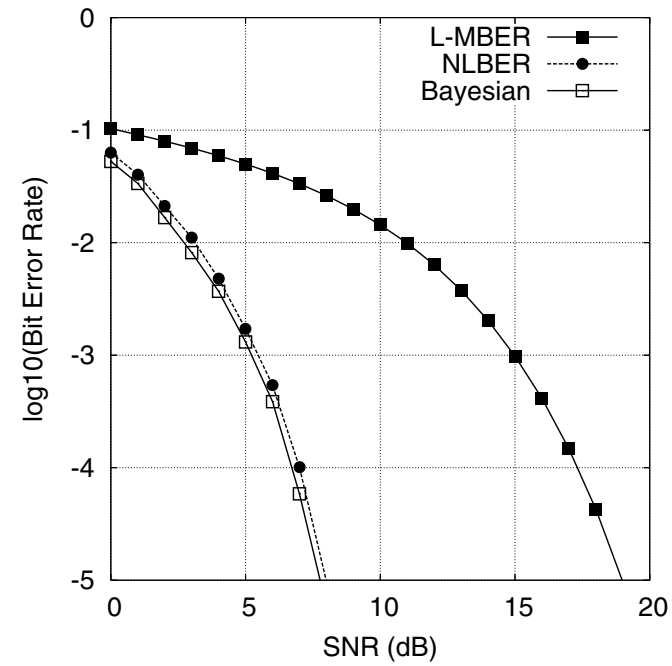

Fig. 6. The desired-user's bit error rate performance for the three-element antenna array supporting five users at the angular positions of Table I. The NLBER-based RBF detector has $n_{c}=16$ symmetric RBF nodes.

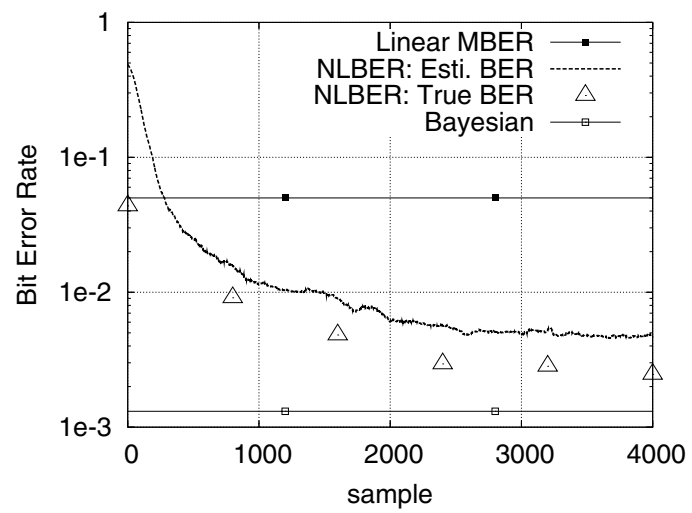

Fig. 7. Learning curve of the NLBER-based RBF detector averaged over 10 runs for the three-element antenna array supporting five users, where $\mathrm{SNR}=$ $5 \mathrm{~dB}$ and the RBF detector has $n_{c}=16$ symmetric RBF nodes.

Example 2. The BER performance of the two benchmarkers, the L-MBER beamformer and the Bayesian detector, are shown in Fig. 6. The size of the Bayesian detector is $N_{s b}=16$. The convergence performance of the NLBER algorithm is characterised in Fig. 7, given $\mathrm{SNR}=5 \mathrm{~dB}$ and $n_{c}=16$. Again, the learning curve of Fig. 7 was averaged over 10 runs, and we used the first 16 data points as the initial RBF centres, set all the initial RBF weights to 0.06 and all the initial RBF variances to $9 \sigma_{n}^{2}$. The two algorithmic parameters of the NLBER algorithm were chosen as step size $\mu=0.4$ and kernel variance $\rho^{2}=4 \sigma_{n}^{2}$. The block size used for estimating the approximated BER $\tilde{P}_{E}(\mathbf{w}(k))$ was $K=800$ with a kernel variance of $\rho^{2}=\sigma_{n}^{2}$. The true BER markers (triangles) in Fig. 7 confirm that the learning curve $\tilde{P}_{E}(\mathbf{w}(k))$ correctly indicated the convergence trend and the algorithm achieved convergence after 3000 samples of training. Given $\mathrm{SNR}=5 \mathrm{~dB}$ and the same initial conditions as before, Fig. 8 illustrates the influence of the number of RBF centres $n_{c}$ on the BER performance of the NLBER-based symmetric RBF detector. Using $n_{c}=16$ symmetric RBF nodes, the performance of the NLBER-based symmetric RBF detector is compared to those

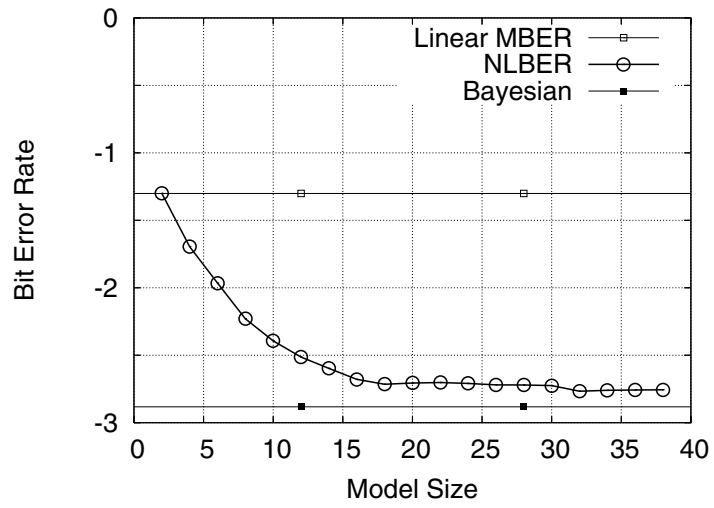

Fig. 8. The influence of the detector's size on the bit error rate performance of the NLBER-based symmetric RBF detector for the three-element antenna array supporting five users, where $\mathrm{SNR}=5 \mathrm{~dB}$.

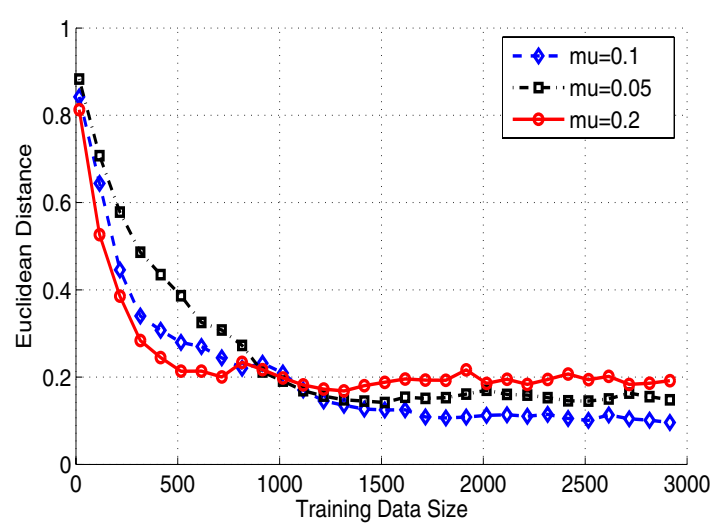

Fig. 9. Learning curve of the clustering-based RBF detector averaged over 5 runs for the three-element antenna array supporting five users, where SNR= $5 \mathrm{~dB}$ and the RBF detector has $n_{c}=16$ symmetric RBF nodes.

of the other two benchmarkers in Fig. 6.

For the clustering-based symmetric RBF detector, again we used the first $N_{s b}$ data points as the initial centres $\mathbf{c}_{i}(0)$ and set all the RBF variances to $2 \sigma_{n}^{2}$. The convergence of the clustering algorithm (17) averaged over 5 runs was illustrated in Fig. 9 for the three values of the step size $\mu_{c}$, where the initial clustering variations $\bar{v}_{i}(0)$ were again set to 0.01 and the adaptive gain for updating the clustering variations was set to $\mu_{v}=0.995$. The BER performance of the clustering-based symmetric RBF detector is depicted in Fig. 10, in comparison with the two benchmarkers. Finally, Fig. 11 illustrates the influence of the detector's size on the BER performance of the clustering-based symmetric RBF detector.

\section{CONCLUSIONS}

A symmetric RBF network has been proposed for nonlinear detection in beamforming, which substantially outperforms previous solutions found in the literature in the challenging scenario of supporting almost twice as many users, as the number of antenna elements in multiple-antenna aided beamforming systems. Two adaptive algorithms, the clustering and NLBER, have been derived for training the symmetric RBF detector. It has been shown using a simulation study that these two algorithms are capable of approaching the optimal Bayesian detection performance. The proposed solution is 


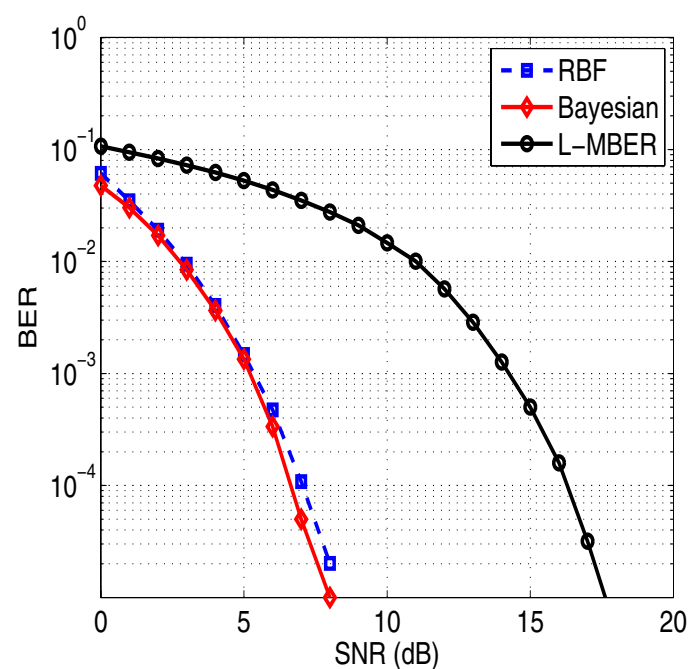

Fig. 10. The desired-user's bit error rate performance for the three-element antenna array supporting five users at the angular positions of Table I. The clustering-based RBF detector has $n_{c}=16$ symmetric RBF nodes.

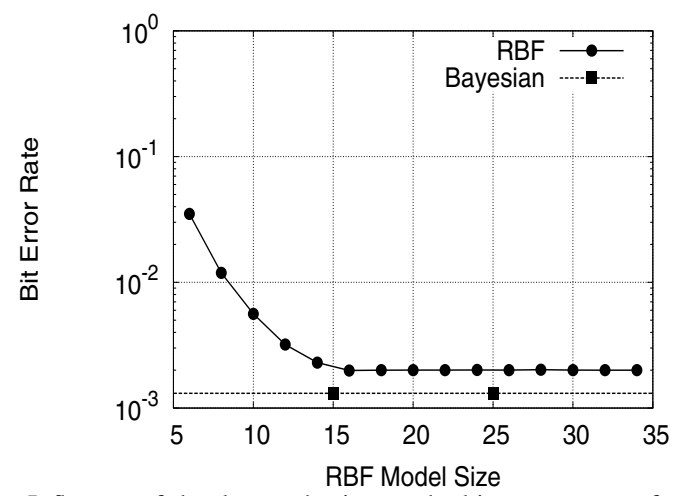

Fig. 11. Influence of the detector's size on the bit error rate performance of the clustering-based symmetric RBF detector for the three-element antenna array supporting five users, where $\mathrm{SNR}=5 \mathrm{~dB}$.

capable of providing an SNR gain in excess of $8 \mathrm{~dB}$ against the powerful linear minimum bit error rate benchmarker, when supporting four users with the aid of two receive antennas or five users employing three receive antenna elements.

\section{ACKNOWLEDGEMENTS}

The financial support of the EU under the auspice of the Newcom project is gratefully acknowledged.

\section{REFERENCES}

[1] J. Litva and T.K.Y. Lo, Digital Beamforming in Wireless Communications. London: Artech House, 1996.

[2] L. C. Godara, "Applications of antenna arrays to mobile communications, Part II: Beam-forming and direction-of-arrival considerations," Proc. IEEE, vol.85, no.8, pp.1193-1245, 1997.

[3] J.H. Winters, "Smart antennas for wireless systems," IEEE Personal Communications, vol.5, no.1, pp.23-27, 1998.

[4] R. Kohno, "Spatial and temporal communication theory using adaptive antenna array," IEEE Personal Communications, vol.5, no.1, pp.28-35, 1998.

[5] G.V. Tsoulos, "Smart antennas for mobile communication systems: benefits and challenges," IEE Electronics and Communications J., vol.11, no.2, pp.84-94, 1999.

[6] P. Vandenameele, L. van Der Perre and M. Engels, Space Division Multiple Access for Wireless Local Area Networks. Boston: Kluwer Academic Publishers, 2001.
[7] J.S. Blogh and L. Hanzo, Third Generation Systems and Intelligent Wireless Networking - Smart Antennas and Adaptive Modulation. Chichester, U.K.: Wiley, 2002.

[8] A. Paulraj, R. Nabar and D. Gore, Introduction to Space-Time Wireless Communications. Cambridge, U.K.: Cambridge University Press, 2003.

[9] A.J. Paulraj, D.A. Gore, R.U. Nabar and H. Bölcskei, "An overview of MIMO communications - A key to gigabit wireless," Proc. IEEE, vol.92, no.2, pp.198-218, 2004.

[10] D. Tse, and P. Viswanath, Fundamentals of Wireless Communication. Cambridge, U.K.: Cambridge University Press, 2005.

[11] D.N.C. Tse and S.V. Hanly, "Linear multiuser receivers: effective interference, effective bandwidth and user capacity," IEEE Trans. Information Theory, vol.45, no.2, pp.641-657, 1999.

[12] S. Chen, N.N. Ahmad and L. Hanzo, "Adaptive minimum bit error rate beamforming," IEEE Trans. Wireless Communications, vol.4, no.2, pp.341-348, 2005.

[13] S. Chen, L. Hanzo, N.N. Ahmad and A. Wolfgang, "Adaptive minimum bit error rate beamforming assisted receiver for QPSK wireless communication," Digital Signal Processing, vol.15, no.6, pp.545-567, 2005.

[14] K. Abend and B.D. Fritchman, "Statistical detection for communication channels with intersymbol interference," Proc. IEEE, vol.58, no.5, pp.779-785, 1970.

[15] S. Chen, S. McLaughlin, B. Mulgrew and P.M. Grant, "Adaptive Bayesian decision feedback equaliser for dispersive mobile radio channels," IEEE Trans. Communications, vol.43, no.5, pp.1937-1946, 1995.

[16] S. Chen, L. Hanzo and A. Wolfgang, "Nonlinear multi-antenna detection methods," EURASIP J. Applied Signal Processing, vol.2004, no.9, pp.1225-1237, 2004.

[17] S. Chen and B. Mulgrew, "Overcoming co-channel interference using an adaptive radial basis function equaliser," Signal Processing, vol.28, no.1, pp.91-107, 1992.

[18] S. Chen, B. Mulgrew and P.M. Grant, "A clustering technique for digital communications channel equalisation using radial basis function networks," IEEE Trans. Neural Networks, vol.4, no.4, pp.570-579, 1993.

[19] A. Wolfgang, S. Chen and L. Hanzo, "Radial basis function network assisted space-time equalisation for dispersive fading environments," Electronics Letters, vol.40, no.16, pp.1006-1007, 2004.

[20] F. Albu and D. Martinez, "The application of support vector machines with Gaussian kernels for overcoming co-channel interference," in Proc. 9th IEEE Int. Workshop Neural Networks for Signal Processing (Madison, WI), Aug.23-25, 1999, p.49-57.

[21] D.J. Sebald and J.A. Bucklew, "Support vector machine techniques for nonlinear equalization," IEEE Trans. Signal Processing, vol.48, no.11, pp.3217-3226, 2000.

[22] S. Chen, A.K. Samingan and L. Hanzo, "Support vector machine multiuser receiver for DS-CDMA signals in multipath channels," IEEE Trans. Neural Networks, vol.12, no.3, pp.604-611, 2001.

[23] S. Chen, S.R. Gunn and C.J. Harris, "The relevance vector machine technique for channel equalization application," IEEE Trans. Neural Networks, vol.12, no.6, pp.1529-1532, 2001.

[24] F. Pérez-Cruz, A. Navia-Vázquez, P.L. Alarcón-Diana and A. ArtésRodrguez, "SVC-based equalizer for burst TDMA transmissions," Signal Processing, vol.81, no.8, pp.1571-1787, 2001.

[25] S. Chen, L. Hanzo and A. Wolfgang, "Kernel-based nonlinear beamforming construction using orthogonal forward selection with Fisher ratio class separability measure," IEEE Signal Processing Letters, vol.11, no.5, pp.478-481, 2004.

[26] L.A. Aguirre, R.A.M. Lopes, G.F.V. Amaral and C. Letellier, "Constraining the topology of neural networks to ensure dynamics with symmetry properties," Physical Review E, vol.69, pp.026701-1-026701-11, 2004.

[27] M. Espinoza, J.A.K. Suykens and B. De Moor, "Imposing symmetry in least squares support vector machines regression," in Proc. Joint 44th IEEE Conf. Decision and Control, and the European Control Conf. 2005 (Seville, Spain), Dec.12-15, 2005, pp.5716-5721.

[28] R.O. Duda and P.E. Hart, Pattern Classification and Scene Analysis. New York: Wiley, 1973.

[29] E. Parzen, "On estimation of a probability density function and mode," The Annals of Mathematical Statistics, vol.33, pp.1066-1076, 1962.

[30] B.W. Silverman, Density Estimation. London, U.K.: Chapman Hall, 1996.

[31] A.W. Bowman and A. Azzalini, Applied Smoothing Techniques for Data Analysis. Oxford, U.K.: Oxford University Press, 1997. 